\title{
Imaginaires archéologiques, MCC-SDARCHETISl
}

\section{Emmanuel Ghesquière}

\section{OpenEdition}

\section{Journals}

Édition électronique

URL : http://journals.openedition.org/rao/969

DOI : $10.4000 /$ rao.969

ISBN : 978-2-7535-1609-0

ISSN : 1775-3732

Éditeur

Presses universitaires de Rennes

Édition imprimée

Date de publication : 31 décembre 2009

ISBN : 978-2-7535-1086-9

ISSN : 0767-709x

\section{Référence électronique}

Emmanuel Ghesquière, "Imaginaires archéologiques, MCC-SDARCHETISI », Revue archéologique de I'Ouest [En ligne], 26 | 2009, mis en ligne le 31 décembre 2011, consulté le 04 décembre 2020. URL : http://journals.openedition.org/rao/969; DOI : https://doi.org/10.4000/rao.969 
céramique peinte grecque fournit une documentation iconographique très riche et on l'a toujours considéré comme un objet de prestige dont l'importance était d'autant plus difficile à cerner qu'elle est rarement mentionnée dans les sources anciennes (M. Vickers). Mais il ne faut pas oublier que la vaisselle des riches était à l'époque d'or et d'argent. Il semble maintenant évident que ce type de poterie était utilisé dans des contextes domestiques relativement modestes. Si la céramique est signifiante, et si elle nous apporte beaucoup pour la connaissance des sociétés traditionnelles et antiques, il faut cependant éviter de lui conférer un statut exagéré.

Ce volume nous apporte donc certaines réponses mais il est surtout riche de questions et de nouvelles perspectives stimulantes pour les études céramologiques.

Marie Tuffreau-Libre Directrice de Recherche au CNRS, UMR 6566

Voisenat, Cl. (dir.), 2008 - Imaginaires archéologiques, MCC-SDARCHETIS, Paris, éd. de la Maison des Sciences de l'Homme, coll. «Ethnologie de la France »; 22, 277 p. (ISSN 0758-5888).

Une fois n'est pas coutume, c'est un ouvrage d'ethnologie que nous vous proposons de découvrir. Écrit sous forme de contributions multiples, il concerne directement ou plus souvent indirectement la discipline archéologique. Comme son titre général l'indique, "Imaginaires archéologiques " rassemble une série de contributions traitant du phénomène de la para-archéologie dans la société du $\mathrm{xx}^{\mathrm{e}}$ siècle. De manière réfléchie et avec du recul, les différents auteurs traitent de tout ce qui concerne les abords de l'archéologie scientifique officielle, non pour la dénigrer de façon hautaine comme c'est généralement le cas de notre communauté scientifique, mais pour l'étudier comme un fait de société en marge de la science officielle, avec tout ce qu'elle interroge sur le passé de notre humanité et la popularisation de mythes qui recueillent souvent davantage d'échos que les faits scientifiques.

Claudie Voisenat introduit ainsi l'ouvrage de façon magistrale par une lecture des temps héroïques de la discipline archéologique, à la fin du xix ${ }^{\mathrm{e}}$ siècle. Celle-ci se cherche et hésite encore entre science et folklore, se heurtant souvent à la superstition des populations rurales. Pour finir, en ce qui concerne les mégalithes, l'auteur retient que les inventaires de ceux-ci, initiés par de grandes campagnes de classement au titre des Monuments historiques, se sont recentrés sur le caractère purement scientifique, ne tenant compte de la dimension mystique populaire que de manière annexe, lorsqu'elle n'est pas totalement ignorée.

Le premier article, de Véronique Moulinié, nous plonge dans l'univers étonnant des propriétaires de sites préhistoriques et plus particulièrement des grottes ornées, de la connaissance qu'ils ont de leur(s) site(s) pour l'avoir si souvent parcouru. La propriété de ces lieux de mémoire les amène généralement à assumer des responsabilités politiques locales, acquérant ainsi une charge d'élite avec l'héritage du site.

Le second nous entraine dans le monde trouble des chasseurs de trésors de Rennes-le-Château, sur les traces de l'abbé Saunière. L'auteur décortique ici l'essence même de la para-archéologie où la recherche prend la forme d'une quête du graal, avec ses mythes, ses signes, ses trésors et ses "chevaliers ", armés qui d'une pioche, qui d'un détecteur de métaux. L'importance de la quête est ici flagrante et dépasse celle de l'invention, considérée comme une malédiction.

Claudie Voisenat nous entraine avec l'article suivant vers l'étude des hétéroclites, archivés avec pour icône le dessin d'une tête coiffée d'un entonnoir. Quelle truculence de découvrir sous la plume de l'auteur ce cortège de farfelus, doux-dingues, cherchant des visages dans les pierres ou les traces de géants et d'extraterrestres. Ces illuminés qui vivent leur passion de manière intense, sûrs de leur fait au point d'entretenir des relations épistolaires sur plusieurs dizaines d'années, n'hésitent pas à contacter ministres et présidents, parfois même à l'international, pour se donner une légitimité. Ils ne semblent vivre que pour leur passion stérile qui les dévore. Pour les archéologues de la DAPA, ce fonds de lettres surréalistes sert peut-être finalement à ramener la discipline scientifique à l'homme et à montrer les limites de celle-ci, science humaine non exacte avant tout.

Suivent ensuite un article de Daniel Fabre traitant de l'étonnant rapport de l'écrivain Georges Bataille avec la grotte de Lascaux et un autre de Lucie Désidéri sur la Gravida, nom donné à une figure féminine d'un bas-relief antique, égérie d'un important cortège d'artistes.

Trois contributions de Jean-Pierre Chambon, Marc Pessin et Michaël Jasmin nous entraînent à la découverte de la civilisation pessinoise, inventée par Marc Pessin, qui crée des œuvres, les enterre afin qu'elles acquièrent un statut ou une nature de témoins archéologiques d'une civilisation antique, puis les exhume et les expose. Cette invention, un des auteurs la compare aux œuvres de Duchamp qui transforme des objets usuels qui acquièrent ainsi un autre statut, ici œuvres d'art, pour Marc Pessin témoins archéologiques. La mise en scène ici de l'enfouissement et de l'exhumation 
prend ici une dimension christique pour se métamorphoser de sculptures actuelles à témoins archéologiques.

L'article de Pierre Lagrange traite du délicat sujet de l'Atlantide, versé actuellement dans le mythe plus ou moins lié à l'âge du Bronze européen. Ici l'auteur traite de la période entre les deux guerres où son étude relevait encore du champ scientifique, où l'on s'interrogeait sur la possible présence quaternaire d'un continent dans l'Atlantique, reliant l'Europe ou l'Afrique aux Amériques. Il s'avère maintenant que ce continent a disparu depuis trop longtemps pour que l'humanité en ait gardé le souvenir, mais dans les années 1920, atlantéens et atlantologues se sont opposés dans leur recher- che sur le continent disparu, dérivant finalement sur les divagations mystiques qui perdurent encore actuellement.

Louvrage s'achève sur une contribution de Gaétano Ciarcia. Il y traite des travaux de l'ethnologue Marcel Griaule en Afrique, traquant les civilisations disparues par un mélange d'ethnologie et d'archéologie et aménageant les résultats suivant sa convenance.

En bref, un livre offrant une lecture différente de l'archéologie, nous renvoyant parfois sa pratique dans un miroir déformé, loin de la discipline scientifique que nous pratiquons. Quoique...

Emmanuel GHESQUiÈRE INRAP Basse-Normandie et UMR 6566-CReAAH

Chouquer, G., 2008 - Traité d'archéogéographie. La crise des récits géohistoriques, Paris, Errance, 199 p. (ISBN 978-2-87772$374-9 ; 24 €)$.

Il est ardu de rendre compte d'une œuvre polémique, et celle de Gérard Chouquer n'échappe pas à la règle. Signe des temps, ce nouvel opus du pape de l'archéogéographie est dédié à une crise et non des moindres, celle des " récits géohistoriques", élaborés par les historiens et géographes historiques modernes sur des bases épistémologiques, dont les fondements relèvent depuis le XviI ${ }^{\mathrm{e}}$ siècle de la philosophie cartésienne puis de l'idéologie républicaine nationale. Entendons par là crise de l'Histoire-Géographie moderne, héritée en dernière instance des idoles $\mathrm{du} \mathrm{xx}^{\mathrm{e}}$ siècle (Marc Bloch, Fernand Braudel...), dans un contexte plus général et tout à fait d'actualité de crise des sciences humaines, ces sciences «molles » qui, par opposition à celles réputes "dures", subissent aujourd'hui les assauts d'une critique sans aucun doute nécessaire, mais souvent dirigée.

Dès la lecture du préambule, la composition et la finalité affichée de l'ouvrage suscitent une première interrogation : ce " traité " d'archéogéographie en est-il bien un? Le dialogue permanent entre la chose et le mot, l'objet et la forme, la question et les réponses composent un développement fourmillant, d'une grande richesse intellectuelle, soutenu par une érudition sans faille. Toutefois, la solution archéogéographique vers laquelle convergent l'ensemble de la démonstration et l'appareil critique ne nous apparaît pas d'une limpide évidence. À travers le prisme d'une critique érudite, souvent austère, émaillée de néologismes colorés (à connotation souvent « anatomique " et / ou " médicale ", tels ces « collecteurs hypertrophiés»), entre le je-ne-sais-quoi et le presque-rien de l'épistémologie contemporaine, s'esquisse un engagement individuel en faveur de l'intérêt collectif à travers la salutaire irruption du présent dans les sciences du passé. Il s'agirait donc davantage à nos yeux d'un essai voire d'un manifeste dont le contenu est avant tout question de regard, induit par les angles et focales retenus par l'auteur, de même que les mots soigneusement choisis et disséqués pour le dire. À ce titre, Gérard Chouquer et ses disciples archéogéographes font montre dans ces pages d'une maîtrise lexicologique remarquable, tout entière employée à la dissection d'un discours dominant dont ils démontent un par un les mécanismes culturels, politiques et idéologiques forgés par le nationalisme identitaire imputé (de façon peut-être un rien caricaturale?) aux « savants » des $\mathrm{XIX}^{\mathrm{e}}$ - $\mathrm{XX}^{\mathrm{e}}$ siècles.

L'exposé du " projet archéogéographique " se fonde sur trois enquêtes, dont l'enseignement est décliné au sein de deux parties. La première enquête est un constat, celui de «la modernité anachronique des objets prémodernes élaborés par les savants et donc de l'inadaptation de ces objets à l'étude des passés lointains ». Le terme " objet " est défini, p. 7, comme « des élaborations plus ou moins fondées, présentées comme des objets historiques avec lesquels on écrit d'ordinaire l'histoire de l'espace-temps " (domaine antique, villa, cité, centuriation, voie romaine, village, etc.). L'auteur propose donc une interrogation de la Modernité qui débouche sur une remise en cause, parfois radicale, des dits objets au regard des réalités qu'ils sont censés définir et condenser. La deuxième enquête aborde le problème de l'historiographie et en particulier celui du foisonnement disciplinaire qui la caractérise, signe d'un "individualisme méthodologique » que $\mathrm{G}$. Chouquer et $\mathrm{M}$. Watteaux dissèquent à travers pas moins d'une cinquantaine d'intitulés, disciplinaires ou thématiques. La troisième enquête propose enfin un cadre théorique et épistémologique à la rénovation des 